\title{
Genetic influences on dynamic complexity of brain oscillations
}

\author{
Andrey P. Anokhin ${ }^{\mathrm{a}, *}$, Viktor Müller ${ }^{\mathrm{b}, \mathrm{c}}$, Ulman Lindenberger ${ }^{\mathrm{b}, \mathrm{c}}$, \\ Andrew C. Heath ${ }^{\mathrm{a}}$, Erin Myers ${ }^{\mathrm{a}}$ \\ ${ }^{a}$ Washington University School of Medicine, Department of Psychiatry, 18 S.Kingshighway, Suite 2T/U, St. Louis, MO 63108, USA \\ ${ }^{\mathrm{b}}$ Center for Lifespan Psychology, Max Planck Institute for Human Development, Berlin, Germany \\ c School of Psychology, Saarland University, Saarbrücken, Germany
}

Received 7 September 2005; received in revised form 23 November 2005; accepted 1 December 2005

\begin{abstract}
Human electroencephalogram (EEG) consists of complex aperiodic oscillations that are assumed to indicate underlying neural dynamics such as the number and degree of independence of oscillating neuronal networks. EEG complexity can be estimated using measures derived from nonlinear dynamic systems theory. Variations in such measures have been shown to be associated with normal individual differences in cognition and some neuropsychiatric disorders. Despite the increasing use of EEG complexity measures for the study of normal and abnormal brain functioning, little is known about genetic and environmental influences on these measures. Using the pointwise dimension (PD2) algorithm, this study assessed heritability of EEG complexity at rest in a sample of 214 young female twins consisting of 51 monozygotic (MZ) and 56 dizygotic (DZ) pairs. In MZ twins, intrapair correlations were high and statistically significant; in DZ twins, correlations were substantially smaller. Genetic analyses using linear structural equation modeling revealed high and significant heritability of EEG complexity: 62-68\% in the eyes-closed condition, and $46-60 \%$ in the eyes-open condition. Results suggest that individual differences in the complexity of resting electrocortical dynamics are largely determined by genetic factors. Neurophysiological mechanisms mediating genetic variation in EEG complexity may include the degree of structural connectivity and functional differentiation among cortical neuronal assemblies.
\end{abstract}

(C) 2005 Elsevier Ireland Ltd. All rights reserved.

Keywords: Electroencephalogram; Complexity; Nonlinear dynamics; Twins; Heritability

Neural activity underlying cognition and behavior is characterized by a high degree of functional differentiation and, at the same time, functional integration achieved through rapid binding of spatially distributed and functionally specialized neuronal groups [32]. To some extent, these fundamental properties of large-scale brain dynamics are reflected in the electroencephalogram (EEG) recorded from the scalp. The EEG results from the summation of postsynaptic activity of a large number of spatially distributed but functionally connected and interacting cortical neurons and neuronal assemblies. Accordingly, the EEG time series has a complex structure reflecting the complexity of the underlying neural generators [16,26]. A greater number of independent processes contributing to the EEG results in a greater complexity of EEG time series [18]. EEG complexity may reflect the number of states of a system resulting from the interaction

\footnotetext{
* Corresponding author. Tel.: +1 314286 2201; fax: +1 3142860092

E-mail address: andrey@matlock.wustl.edu (A.P. Anokhin).
}

among its elements, with higher complexity reflecting a larger number of separable oscillatory networks [32].

In recent years, cortical oscillations have been increasingly viewed from the perspective of nonlinear dynamic systems theory, which investigates complex, aperiodic systems capable of self-organization $[9,26]$. The behavior of complex systems can be quantified with dimensionality measures that reflect the degree of their dynamic complexity $[16,26]$. In the case of EEG, complexity refers to the dimension of the reconstructed statespace attractor of the EEG time series. Simulation studies have shown that the correlation dimension of finite time series generated by multiple oscillators monotonously increases with the number of oscillators, suggesting that dimensional complexity of the EEG can be indicative of the number of independently oscillating neuronal networks in the cortex that give rise to the EEG signal [18]. Dynamic complexity may be associated with competition among neuronal cell assemblies that do not settle at a common frequency $[17,18]$. The question whether dimensional complexity represents a measure of nonlinear dynamics, 
that is, chaotic behavior of underlying neuronal populations, is still a matter of discussion [26,31]. Mathematically, the behavior of nonlinear dynamic systems cannot be expressed as a linear function of its descriptors and does not obey the principle of superposition (e.g., the system is not equal to the sum of its parts). In this sense, a nonlinear system contains information not captured by linear autoregressive processes and is thus, different from a (linear) stochastic system with unlimited degrees of freedom. However, regardless of whether EEG is strictly nonlinear or not, EEG complexity measures can provide useful information about the dimensionality of underlying neuronal dynamics.

Measures of EEG complexity vary systematically within individuals as a function of functional state, for instance, as a result of task manipulation. Generally, complexity appears to be higher in tasks requiring greater diversity of neural representations, that is, concurrent activation of multiple associative neural networks that oscillate at different frequencies $[17,19,28]$. In contrast, structured tasks requiring selective and focused attention result in lower complexity, presumably reflecting suppression of redundant activity [17,19,28]. Individual differences in EEG complexity correlate with differences in cognitive functioning such as general intelligence [2], suggesting that EEG complexity is a trait-like measure that is indicative of important individual differences in brain dynamics.

It also has been suggested [5] that dysregulation of a fine-tuned balance between chaotic and non-chaotic neuronal dynamics underlying behavior can be an important factor in various forms of psychopathology. This hypothesis has received increasing support by evidence indicating significant abnormalities in regional or global EEG complexity in patients with neuropsychiatric disorders compared to controls. Examples include Alzheimer's disease [12,13], Parkinson's disease [22,31], schizophrenia [8,15,25], post-traumatic stress disorder [7], and mania [4]. The clinical and diagnostic utility of complexity measures in these and related contexts remains to be explored.

In sum, EEG complexity is a promising indicator of neuronal dynamics that may contribute to a better understanding of the mechanisms underlying both normal and abnormal brain function. In particular, disturbed cortical oscillatory dynamics as reflected in dimensionality measures may serve as a biological marker of functional brain abnormalities in some neuropsychiatric disorders.

The relative roles of genetic and environmental factors in the etiology of individual differences in EEG complexity are not known. If EEG complexity was found to be heritable, it may prove to be a useful indicator of genetically transmitted characteristics of cortical dynamics. Thus, the purpose of this study was to estimate the relative contribution of genetic factors to individual variability in EEG complexity (heritability) using quantitative EEG recordings from twins. The classical twin approach based on the comparison of monozygotic (MZ) and dizygotic (DZ) twins provides a powerful tool for partitioning genetic and environmental sources contributing to the phenotypic variation in a quantitative trait [27]. To the best of our knowledge, this is the first study to estimate heritability of the EEG's dynamic complexity.
Research participants were 214 young adult female twins including $51 \mathrm{MZ}$ and $56 \mathrm{DZ}$ pairs (age 18-28 years, $M=21.7$, S.D. $=2.8$ ) recruited from the general population through a twin registry. Individuals with a history of serious head trauma, known diagnoses of neurological disorders, or currently using psychoactive medication were excluded. Zygosity was determined using a standard interview administered to both twins. The reliability of zygosity diagnosis by questionnaire has been demonstrated in previous studies [14]. In addition, interviews with parents and blood test data were available for about $15 \%$ of the pairs. All experiments involving humans were conducted in accordance with the Declaration of Helsinki. The study was approved by Washington University Institutional Review Board, and informed consent was obtained from all participants.

The resting EEG recording was performed twice during the experimental session that also included other psychophysiological paradigms described elsewhere. Participants were seated in a recliner chair and instructed to take a comfortable position, relax, sit still and avoid major movements and muscle tension. Each resting recording consisted of four 1-min EEG segments recorded with eyes closed or open in an alternating sequence (EC-EO-EC-EO). During the eyes-open condition, the participant was instructed to maintain her gaze on the blank computer screen and to avoid major eyes and head movements. The EEG was recorded from 19 scalp locations according to the 10-20 system using an elastic cap with silver-chloride electrodes (Quik-Cap, Neuroscan, Inc.) and a ground electrode on the forehead, with high- and low-pass filters set at 0.05 and $70 \mathrm{~Hz}$, respectively. The left mastoid served as reference, and an averaged mastoid reference was digitally computed off-line using the right mastoid recording as a separate channel. The EEG signal was digitized at a rate of 1000 samples per second. A correction for ocular artifacts such as blinks and saccades present in the eyes-open condition was performed using a regression procedure [29]. After screening for other artifacts (movement, poor electrode contact, electromyographic activity) the EEG signals were subjected to further analyses. However, no clinical evaluation of EEG recordings was performed and no further exclusions were made based on possible EEG abnormalities.

The pointwise correlation dimension (PD2) was calculated using the Dataplore software package (Datan Software and Analysis GmbH, Teltow, Germany). For the estimation of PD2, this package uses Skinner's [30] algorithm and computes the PD2 based on the following formula: $P D 2(i)=\log C(r, i) / \log (r)$, with the pointwise correlation integral computed as:

$C(r, i)=\frac{1}{N-1} \sum_{j=0 ; j \neq i}^{N-1} \theta\left(r-\left\|\hat{x}_{i}-\hat{x}_{j}\right\|\right)$

where $r$ denotes the radius of a phase-space neighborhood around $x ; \hat{x}_{i}$ and $\hat{x}_{j}$ are the phase-space coordinates, with a delay of $\tau, N$ is the length of the signal, and $\theta$ is the Heavyside function, given by:

$\theta(x)=\left\langle\begin{array}{ll}0 & \text { if } x<0 \\ 1 & \text { if } x \geq 0\end{array}\right.$ 
PD2 returns the dimension of an attractor of the reconstructed time series, thereby yielding a measure of the system's dynamic complexity. The PD2 algorithm rejects unsuitable estimates that do not result in linear scaling or clear convergence during dimensionality determination and is therefore, well-suited to examine non-stationary time series, and is applicable to EEG and other biological signals (reviewed in [9]). Others [20] have suggested that the PD2 algorithm is more accurate than other algorithms used to estimate the correlation dimension, such as D2 [11] and the pointwise scaling dimension D2i or PWSD [10], which assume data stationarity.

Time series were reconstructed with an embedding dimension of 24 and a time delay of $8 \mathrm{~ms}$. The choice of this embedding dimension was guided by the following considerations: if the embedding dimension is too low, the phase space of the reconstructed time series cannot be fully unfolded [11]; if the dimension is too high, it will amplify the effects of high-dimensional noise. Preliminary analyses of data using different settings indicated that the number of estimated dimensions began to saturate at this point. Thus, the embedding dimension of 24 appeared sufficiently close to an optimal representation of the dynamic properties of the time series under investigation. The time delay, or tau value, was determined by the first minimum in the autocorrelation function of the time series according to common practice [9]. This procedure effectively prevents high correlation between consecutive data points and leads to a more realistic reconstruction of the phase space and correspondingly better determination of the system's dynamic properties. Scanning resolution or skip interval was equal to 64 , that is, the PD2-value was calculated for each 64-th sample as a moving reference point in the sequence. PD2 was calculated for artifact-free $8192 \mathrm{~ms}$ EEG segments (8192 data points) and then averaged over the 125 determined PD2 values in the sequence. Three such artifact-free segments were selected from each of the two 1-min segments in each of the two resting recordings. Thus, a total of $12 \mathrm{seg}$ ments were used to characterize each of the two conditions (eyes closed versus eyes open) in each subject (three segments $\times$ two 1 -min segments $\times$ two recordings), except for six participants in the rest 1 condition and nine subjects in the rest 2 condition, who contributed fewer segments due to artifacts. EEG analyses were performed blindly with respect to the subjects' zygosity status.

Since preliminary analyses indicated strong positive correlations among PD2 measures at individual electrodes, a data reduction was performed using principal component analysis on PD2 measures derived from all 19 electrodes. Individual scores on the first principal components were used in further analyses as a global measure of EEG complexity, in addition to PD2 measures for individual electrodes.

To estimate the relative contribution of genetic and environmental sources to the total phenotypic variance of the PD2 measures (heritability), we performed a biometrical genetic analysis using a model-fitting approach that has become standard in twin genetic research [24,27]. Analysis of the population distributions of PD2 measures in different cortical areas indicated that they were reasonably close to normal. Intrapair twin correlations and variance-covariance matrices were computed separately for
MZ and DZ twins. Linear structural equation models were fitted to empirical variance-covariance matrices using the $\mathrm{Mx}$ package specifically developed to model genetically informative data [23]. These models assume that phenotypic variance arises from the following factors: additive genetic influences (A), non-additive genetic influences (D) or environmental influences shared by family members (C), and individually unique (unshared) environmental influences (E). Path coefficients corresponding to these factors were estimated using maximum likelihood statistics, and a $\chi^{2}$ statistic was used to assess the goodness-of-fit of each model, where low $\chi^{2}$ values indicate a good fit. In addition, different models were compared using Akaike's information criterion (AIC, computed as $\chi^{2}-2$ d.f.) that provides a combined measure of goodness-of-fit and parsimony of a given model. The model with the lowest AIC (i.e., largest negative) was considered best fitting. Heritability was estimated as the percentage of the total variance of the trait attributable to genetic factors; in addition, asymmetric $95 \%$ confidence intervals of the estimate were computed. A detailed description of the model-fitting approach and assessment of heritability can be found elsewhere [24,27]. Since regional differences in the results of genetic analyses were minimal, results are presented for the most representative electrode locations $\mathrm{Fz}$, $\mathrm{Cz}$, and $\mathrm{Pz}$ (frontal, central, and parietal midline electrodes, respectively) as well as for the PCA-based global PD2 measure.

Average PD2 values and their dependence on condition (eyes closed versus eyes open) were similar to those described in a previous study using the same PD2 estimation algorithm for the analysis of magnetoencephalographic (MEG) recordings [21], suggesting that the PD2 measure is indicative of some fundamental properties of neuronal cortical dynamics that may generalize across measurement methods. The first principal component accounted for $62.8 \%$ of the total variance of the PD2 measures, with positive loadings of measures from all electrode locations. Analysis of variance showed no significant differences between $\mathrm{MZ}$ and DZ twins with respect to means and variances of the PD2 measures and age. A visual inspection of the EEGs with the lowest and highest $10 \%$ of PD2 values did not suggest that extreme PD2 values reflected clinically abnormal EEG patterns.

Intrapair correlations computed for MZ twins were significant for all PD2 measures, ranging from 0.46 to 0.69 . In contrast, DZ twin correlations were substantially lower and only about half of them were statistically significant. On average, DZ correlations were about half as large as MZ correlations, suggesting a genetic influence.

A formal genetic analysis performed using linear structural equation modeling showed significant effects of additive genetic factors on all PD2 measures. The AE model including additive genetic (A) and individual environmental (E) factors showed a good fit for all measures with significant twin correlations. For some of the measures, $\mathrm{MZ}$ correlations were less than twice the corresponding DZ correlations, suggesting a possible contribution of shared environmental factors. However, the inclusion of the shared environmental component (C) did not lead to a significant improvement of fit, and the confidence intervals for the estimate of $\mathrm{C}$ included zero. Since it is standard in model-fitting 
Table 1

Twin intrapair correlations and estimates of genetic and environmental variance components for PD2 scores

\begin{tabular}{|c|c|c|c|c|c|c|c|c|}
\hline Trait & Mean \pm S.D. & $r_{\mathrm{MZ}}(n=51)$ & $r_{\mathrm{DZ}}(n=56)$ & $a^{2}(95 \% \mathrm{CI})$ & $e^{2}(95 \% \mathrm{CI})$ & $\chi^{2}($ d.f. $=4)$ & $p$ & AIC \\
\hline \multicolumn{9}{|c|}{ PD eyes closed } \\
\hline $\mathrm{Fz}$ & $5.39 \pm .44$ & $.64^{* * * *}$ & $.29^{*}$ & $.62(.44-.75)$ & $.38(.25-.56)$ & 2.80 & .59 & -5.20 \\
\hline $\mathrm{Cz}$ & $5.37 \pm .43$ & $.69^{* * *}$ & $.35^{* *}$ & $.68(.52-.79)$ & $.32(.21-.48)$ & .77 & .94 & -7.23 \\
\hline $\mathrm{Pz}$ & $5.29 \pm .49$ & $.65^{* * *}$ & $.43^{* *}$ & $.65(.50-.76)$ & $.35(.24-.50)$ & 1.44 & .84 & -6.56 \\
\hline Factor 1 & $0.0 \pm 1.0$ & $.64^{* * *}$ & $.33^{*}$ & $.65(.48-.76)$ & $.35(.24-.52)$ & .59 & .96 & -7.41 \\
\hline \multicolumn{9}{|c|}{ PD eyes open } \\
\hline $\mathrm{Fz}$ & $5.58 \pm .46$ & $.50^{* * *}$ & .24 & $.48(.27-.65)$ & $.52(.35-.73)$ & 2.81 & .59 & -5.19 \\
\hline $\mathrm{Cz}$ & $5.52 \pm .44$ & $.56^{* * *}$ & $.32^{*}$ & $.55(.36-.69)$ & $.45(.31-.64)$ & 1.15 & .89 & -6.85 \\
\hline $\mathrm{Pz}$ & $5.59 \pm .47$ & $.46^{* *}$ & .25 & $.46(.24-.63)$ & $.54(.37-.76)$ & 1.08 & .90 & -6.92 \\
\hline Factor 1 & $0.0 \pm 1.0$ & $.63^{* * *}$ & .17 & $.60(.40-.74)$ & $.40(.26-.60)$ & 2.07 & .72 & -5.94 \\
\hline
\end{tabular}



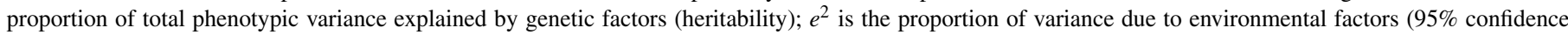

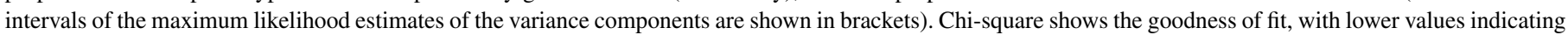
a better fit; AIC is Akaike's information criterion.

* Significance levels, $p<0.05$.

** Significance levels, $p<0.01$.

*** Significance levels, $p<0.001$.

analyses to accept the simpler (more parsimonious) model when two models explain the observed data equally well, parameter estimates are presented for the AE model (Table 1). The percentage of variance in PD2 measures accounted for by genetic factors (heritability) ranged from 62 to $68 \%$ for the eyes-closed condition and from 0.46 to $0.60 \%$ for the eyes-open condition. The PD2 measures in the central scalp region $(\mathrm{Cz})$ showed the highest heritability estimates.

Results of the present study suggest substantial genetic influences on individual differences in PD2, a measure of the EEG's dynamic complexity. To our knowledge, this study provides the first evidence for heritability of this measure of brain electric activity. The strongest evidence for genetic influences was obtained for PD2 assessed in posterior regions of the cortex, which is consistent with heritability estimates for the EEG power spectrum [34].

The finding of substantial heritability of cortical dimensional complexity raises questions about the neuromorphological characteristics and neurophysiological mechanisms that mediate genetic influences on PD2. The complexity of electrocortical dynamics as assessed by PD2 may indicate the complexity of the underlying system of neural generators, that is, the number of simultaneously oscillating and relatively independent cortical cell assemblies [18]. This, in turn, may reflect the degree of differentiation of inter-neuronal connectivity in the cortex. A larger number of simultaneously active and relatively independent distributed neuronal networks can give rise to an EEG signal with higher degree of dynamic complexity. On the other hand, high complexity may also indicate an excessive activation of neuronal networks due to reduced inhibitory self-regulation of cortical activity. Previous studies $[33,34]$ have demonstrated substantial genetic influences on EEG power and coherence, a purported index of functional connectivity between neuronal activity in different cortical regions. Since coherence and dynamic complexity show a significant inverse relationship [2], both measures can reflect the degree of integration versus differentiation of cortical neuronal assemblies. The extent to which these two measures of cortical dynamics are influenced by the same or different set of genes remains to be explored.

Alterations in EEG complexity measures have been reported in some neuropsychiatric disorders including schizophrenia $[8,15,25]$. The present findings of high heritability of PD2 measures suggest that EEG complexity measures may help in identifying putative neurophysiological abnormalities mediating genetic liability to neuropsychiatric disorders.

EEG complexity is believed to reflect the diversity of the "repertoire" of neural activity patterns, or differentiated neural states [32] that in turn may depend on the number of simultaneously oscillating neural networks [18]. In a previous study we have shown that EEG complexity increases with age [1], suggesting a greater diversity of spontaneously activated neuronal networks. This functional interpretation of EEG complexity is corroborated by evidence from neuroimaging studies [6] suggesting that aging-related deficits in the performance on memory tasks may be related to nonselective recruitment of brain regions and reduced ability to suppress spontaneous activation of representations that are irrelevant to the task at hand [3]. In another study [22], increased EEG complexity was associated with the disturbances in Parkinson's disease resulting from the recruitment of superfluous cortical networks due to failing inhibition of alternative motor programs in the striatum. Thus, available data suggest that EEG complexity is indicative of important properties of functional organization of cortical activity, including its impairments in certain psychiatric disorders and in normal aging. However, further work is needed for a better understanding of the functional significance of this measure.

Our results indicate substantial genetic influences on individual differences in PD2. The specific genes that affect electrocortical complexity as well as the major neurophysiological mechanisms mediating this genetic influence still need to be determined. In this study, individuals with known neurological disorders were excluded at initial screening. However, in order to ensure that the sample is representative of the entire continuum of EEG variability in the population, no further attempts were 
made to exclude participants on the basis of "abnormal" EEG features. Therefore, heritability of PD2 measures may reflect genetic variation in the "normal" range as well as EEG variation related to genetic liability to a broad spectrum of neuropsychiatric disorders existing in the population.

Some limitations of the present study need to be acknowledged. First, the study included young adult female subjects only, and it remains to be seen in future studies whether heritability of dynamic cortical complexity varies as a function of age group, gender, and their interaction. Second, the study was limited to resting EEG only, and it remains to be determined whether the finding of high heritability extends to EEG recorded under other conditions, such as various cognitive tasks or different emotional states. Next, it remains unclear whether genetic influences estimated here are specific to PD2 or shared with other characteristics of electrocortical activity. Studies involving conjoined multivariate analyses of other characteristics such as spectral power, coherence, and event-related activity are needed in order to clarify the "genetic architecture" of brain dynamics. Another limitation concerns the interpretation of PD2 as a nonlinear measure, as some recent studies have questioned the extent of nonlinearity in EEG signals. However, the correlation dimension can be used as a relative, generic estimate of dynamic complexity of a time series regardless of the source of the complexity (linear, nonlinear or stochastic; see [26]). Finally, because EEG recordings were not evaluated by a clinical neurophysiologist, it remains unclear whether PD2 might be related to organic CNS abnormalities. Furthermore, to ensure that the sample is representative of the entire spectrum of EEG variability in the general population, individuals with psychiatric disorders were not excluded. Since many disorders are fairly common in this age group (e.g. history of depression or substance use disorders), it remains to be determined whether such disorders can partially account for genetic variance in PD2 and, consequently, whether PD2 can serve as an endophenotype (marker of genetic risk) for such disorders.

In conclusion, this study presents the first evidence for heritability of dynamic complexity of human electrocortical activity. We suggest that PD2 can serve as indicator of genetically transmitted individual differences in functional organization of the cerebral cortex.

\section{Acknowledgements}

This work was supported in part by grants DA00421-02 from the National Institute on Drug Abuse, 5P50 AA11998-03 from the National Institute on Alcohol Abuse and Alcoholism (Missouri Alcoholism Research Center), and FOR-448 from the Deutsche Forschungsgemeinschaft (DFG). Parts of this article were prepared while the first author was a visiting scientist at the Max Planck Institute for Human Development, Berlin, Germany.

\section{References}

[1] A.P. Anokhin, N. Birbaumer, W. Lutzenberger, A. Nikolaev, F. Vogel, Age increases brain complexity, Electroencephalogr. Clin. Neurophysiol. 99 (1996) 63-68.
[2] A.P. Anokhin, W. Lutzenberger, N. Birbaumer, Spatiotemporal organization of brain dynamics and intelligence: an EEG study in adolescents, Int. J. Psychophysiol. 33 (1999) 259-273.

[3] L. Backman, O. Almkvist, J. Andersson, A. Nordberg, B. Winblad, R. Reineck, B. Langstrom, Brain activation in young and older adults during implicit and explicit retrieval, J. Cogn. Neurosci. 9 (1997) 378-391.

[4] B. Bahrami, R. Seyedsadjadi, B. Babadi, M. Noroozian, Brain complexity increases in mania, Neuroreport 16 (2005) 187-191.

[5] N. Birbaumer, H. Flor, W. Lutzenberger, T. Elbert, Chaos and order in the human brain, Electroencephalogr. Clin. Neurophysiol. Suppl. 44 (1995) 450-459.

[6] R.L. Buckner, Memory and executive function in aging and AD: multiple factors that cause decline and reserve factors that compensate, Neuron 44 (2004) 195-208.

[7] J.H. Chae, J. Jeong, B.S. Peterson, D.J. Kim, W.M. Bahk, T.Y. Jun, S.Y. Kim, K.S. Kim, Dimensional complexity of the EEG in patients with posttraumatic stress disorder, Psychiatry Res. 131 (2004) 79-89.

[8] T. Elbert, W. Lutzenberger, B. Rockstroh, P. Berg, R. Cohen, Physical aspects of the EEG in schizophrenics, Biol. Psychiatry 32 (1992) 595-606.

[9] T. Elbert, W.J. Ray, Z.J. Kowalik, J.E. Skinner, K.E. Graf, N. Birbaumer, Chaos and physiology: deterministic chaos in excitable cell assemblies, Physiol. Rev. 74 (1994) 1-47.

[10] J.D. Farmer, E. Ott, J.A. Yorke, Dimension of chaotic attractors, Physica D7 (1983) 153-180.

[11] P. Grassberger, I. Procaccia, Measuring the strangeness of strange attractors, Physica D9 (1983) 189-208.

[12] B. Jelles, J.H. van Birgelen, J.P. Slaets, R.E. Hekster, E.J. Jonkman, C.J. Stam, Decrease of non-linear structure in the EEG of Alzheimer patients compared to healthy controls, Clin. Neurophysiol. 110 (1999) $1159-1167$.

[13] J. Jeong, EEG dynamics in patients with Alzheimer's disease, Clin. Neurophysiol. 115 (2004) 1490-1505.

[14] J. Kasriel, L. Eaves, The zygosity of twins: further evidence on the agreement between diagnosis by blood groups and written questionnaires, J. Biosoc. Sci. 8 (1976) 263-266.

[15] D.J. Kim, J. Jeong, J.H. Chae, S. Park, S. Yong Kim, H. Jin Go, I.H. Paik, K.S. Kim, B. Choi, An estimation of the first positive Lyapunov exponent of the EEG in patients with schizophrenia, Psychiatry Res. 98 (2000) 177-189.

[16] W. Lutzenberger, N. Birbaumer, H. Flor, B. Rockstroh, T. Elbert, Dimensional analysis of the human EEG and intelligence, Neurosci. Lett. 143 (1992) $10-14$.

[17] W. Lutzenberger, T. Elbert, N. Birbaumer, W.J. Ray, H. Schupp, The scalp distribution of the fractal dimension of the EEG and its variation with mental tasks, Brain Topogr. 5 (1992) 27-34.

[18] W. Lutzenberger, H. Preissl, F. Pulvermuller, Fractal dimension of electroencephalographic time series and underlying brain processes, Biol. Cybern. 73 (1995) 477-482.

[19] M. Molle, L. Marshall, W. Lutzenberger, R. Pietrowsky, H.L. Fehm, J. Born, Enhanced dynamic complexity in the human EEG during creative thinking, Neurosci. Lett. 208 (1996) 61-64.

[20] M. Molnar, J.E. Skinner, V. Csepe, I. Winkler, G. Karmos, Correlation dimension changes accompanying the occurrence of the mismatch negativity and the P3 event-related potential component, Electroencephalogr. Clin. Neurophysiol. 95 (1995) 118-126.

[21] V. Muller, N. Birbaumer, H. Preissl, C. Braun, G. Mayer-Kress, F. Lang, Effects of hydration and hyperventilation on cortical complexity, Exp. Brain Res. 150 (2003) 341-355.

[22] V. Muller, W. Lutzenberger, F. Pulvermuller, B. Mohr, N. Birbaumer, Investigation of brain dynamics in Parkinson's disease by methods derived from nonlinear dynamics, Exp. Brain Res. 137 (2001) 103-110.

[23] M.C. Neale, S.M. Boker, G. Xie, H.H. Maes, Mx: Statistical Modeling, sixth ed., Department of Psychiatry, 2002.

[24] M.C. Neale, L.R. Cardon, Methodology for Genetic Studies of Twins and Families, Kluwer Academic Publishers, 1992

[25] M.P. Paulus, D.L. Braff, Chaos and schizophrenia: does the method fit the madness? Biol. Psychiatry 53 (2003) 3-11. 
[26] W.S. Pritchard, D.W. Duke, Measuring "chaos" in the brain: a tutorial review of EEG dimension estimation, Brain Cogn. 27 (1995) 353-397.

[27] F.V. Rijsdijk, P.C. Sham, Analytic approaches to twin data using structural equation models, Brief Bioinform. 3 (2002) 119-133.

[28] H.T. Schupp, W. Lutzenberger, N. Birbaumer, W. Miltner, C. Braun, Neurophysiological differences between perception and imagery, Brain Res. Cogn. Brain Res. 2 (1994) 77-86.

[29] H.V. Semlitsch, P. Anderer, P. Schuster, O. Presslich, A solution for reliable and valid reduction of ocular artifacts, applied to the P300 ERP, Psychophysiology 23 (1986) 695-703.

[30] J.E. Skinner, M. Molnar, T. Vybiral, M. Mitra, Application of chaos theory to biology and medicine, Integr. Physiol. Behav. Sci. 27 (1992) 39-53.
[31] C.J. Stam, B. Jelles, H.A. Achtereekte, S.A. Rombouts, J.P. Slaets, R.W. Keunen, Investigation of EEG non-linearity in dementia and Parkinson's disease, Electroencephalogr. Clin. Neurophysiol. 95 (1995) 309317.

[32] G. Tononi, G.M. Edelman, Consciousness and complexity, Science 282 (1998) 1846-1851.

[33] C.E. van Beijsterveldt, P.C. Molenaar, E.J. de Geus, D.I. Boomsma, Genetic and environmental influences on EEG coherence, Behav. Genet. 28 (1998) 443-453.

[34] C.E. van Beijsterveldt, G.C. van Baal, Twin and family studies of the human electroencephalogram: a review and a meta-analysis, Biol. Psychol. 61 (2002) 111-138. 